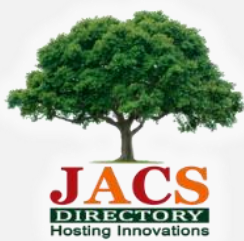

Journal of Nanoscience and Technology

\title{
Hafnium Oxide/Graphene Oxide Nanocomposites as Efficient Supercapacitor Electrodes
}

\author{
M. Jayavel ${ }^{1,2}$, N. Ramalakshmi1 ${ }^{1,}$, S. Arul Antony ${ }^{1}$, J. Venkatachalam ${ }^{3}$ \\ ${ }^{1}$ Department of Chemistry, Presidency College (Autonomous), Chennai - 600 005, Tamil Nadu, India. \\ ${ }^{2}$ Department of Chemistry, Meenakshi College of Engineering, Chennai - 600 078, Tamil Nadu, India. \\ ${ }^{3}$ Department of Physics, Meenakshi College of Engineering, Chennai - 600 078, Tamil Nadu, India.
}

\section{A R T I C L E DETAILS}

\section{Article history:}

Received 03 May 2018

Accepted 13 May 2018

Available online 17 May 2018

\section{Keywords:}

Hafnium Oxide $\left(\mathrm{HfO}_{2}\right)$

Graphene Oxide

Supercapacitor

Cyclic Voltammetry

\begin{abstract}
A B S T R A C T
This paper reports on the preparation of graphene oxide (GO) modified with hafnium oxide (HO) nanoparticles composites by a simple mixing and their use as efficient electrode in electrochemical supercapacitors. The technique relies on simple mixing the aqueous solutions of hafnium oxide (HO) nanoparticles and graphene oxide (GO) at different ratios of GO content $(20,30$ and $40 \mathrm{wt} \%)$. The morphological studies, chemical composition and electrochemical behavior of the resulting $\mathrm{HO} / \mathrm{GO}$ nanocomposites were investigated by using UV/vis spectrometry, X-Ray Diffraction (XRD), scanning electron microscope (SEM), transmission electron microscopy (TEM) and electrochemical respectively. The electrochemical performance, including the capacitive behavior of the $\mathrm{HO} / \mathrm{GO}$ nanocomposites were investigated by cyclic voltammetry and galvanostatic charge-discharge curves. The HO/GO nanocomposites ( $40 \mathrm{wt} \%$ ) ratio exhibited the best performance with a specific capacitance of $268 \mathrm{Fg}^{-1}$ at a scan rate $5 \mathrm{mVs}^{-1}$ in $1 \mathrm{M} \mathrm{H}_{2} \mathrm{SO}_{4}$ and it shows excellent long-term cycle stability.
\end{abstract}

\section{Introduction}

Over the past few years, the growing demand for portable systems and hybrid electrical vehicles requiring high power in short-time pulses, the electrochemical capacitors are gaining the increasing interest because of the up-coming depletion of fossil fuels. Carbon materials such as activated carbon, mesoporous carbon and carbon nanotubes usually display good stability, but the capacitance values are limited by the microstructures in the materials $[1,2]$. Graphene, a two-dimensional all sp² hybridized carbon with unique electronic and mechanical properties, has received a rapidly growing interests as material in supercapacitors [3].

The metal oxide/graphene nanocomposite electrodes shows a better capacitive performance through a combined effect from pseudo capacitance (metal oxide) and electric double layer capacitance (graphene) [4]. $\mathrm{HfO}_{2}$ being the metal oxide group can also be expected to enhance the capacitance when incorporate with graphene oxide. To study the potential of graphene based nanocomposites for supercapacitor applications, different approaches have been considered. The graphene based electrochemical double-layer capacitor with specific capacitance values of $135 \mathrm{Fg}^{-1}$ in aqueous electrolytes is the first report using chemically reduced graphene oxide, synthesized through hydrazine reduction of graphene oxide (GO) [5].

Graphene oxide (GO), one of the most important derivatives of graphene, is characterized by a layered structure with oxygen functional groups bearing on the basal planes and edges [6-8]. Metal oxides depending upon their structural, geometries, and electronic structure play a very significant role in areas of chemistry, physics, biology and material sciences [9]. Supercapacitors in $21^{\text {st }}$ century had attracted more in research interest as an auxiliary and clean source of power and energy [10].

First and third row transition metal oxides seems to be especially attractive as cathode materials in electrochemical energy storage systems due to their favorable electrochemical and solid-state properties. With the aspect of their low price, rich abundance, low toxicity, and diverse oxidation states many transition metal oxide nanoparticles alone and with carboneous filler have got special attention for supercapacitor applications [11-15]. GO provides an effective transportation of ions onto the material surface due to their large accessible surface area, thus accomplishing high electric-double layer capacitance in aqueous electrolytes [16].

The electronic properties of the metal oxide are effected by particle size of material, the quantum size or confinement effects are produced in nanostructured materials which essentially arise from the presence of discrete, atom like electronic state, and also nanosized material is observed to have small band gap in its lattice. Thus the metal oxide nanoparticles can exhibit unique physical and chemical properties due to their limited size and a high density of corner or edge surface sites so metal oxide materials exhibit ionic or mixed ionic/electronic conductivity and are influenced by the nanostructure of the material [17]

Generally, there are two types of supercapacitors based on the electrode materials: (i) some redox active materials that use fast reversible redox reactions at the surface of active materials, which is known as the pseudocapacitance and (ii) high surface area, inert and conductive materials that store and release energy by nanoscopic charge separation at the electrochemical interface between an electrode and an electrolyte [18-21]. The carbonaceous nanocomposites (like CNTs, active carbon and graphene) are commonly studied as electrodes for electrochemical double layer capacitors; while transition metal oxides, including $\mathrm{HO}$, are promising material for pseudocapacitors. The introduction of GO into metal oxides can modify their chemical and physical properties and enhance the activity of supercapacitor. However, to the best of our knowledge, there are no earlier literature on $\mathrm{HO} / \mathrm{GO}$ nanocomposite as efficient supercapacitor.

In the present scenario, we report on the preparation of $\mathrm{HO} / \mathrm{GO}$ composites using the simple mixing with different ratios of GO content $(20$, 30 and $40 \mathrm{wt} \%$ ) gave the corresponding nanocomposites with enhanced electrochemical properties. The HO/GO nanocomposites have been successfully used as electrodes in supercapacitors with a maximum specific capacitance of $268 \mathrm{Fg}^{-1}$ in $1 \mathrm{M} \mathrm{H}_{2} \mathrm{SO}_{4}$ at a scan rate of $5 \mathrm{mVs}^{-1}$.

\section{Experimental Methods}

\subsection{Materials}

Graphite powder, hafnium tetrachloride $\left(\mathrm{HfCl}_{4}\right)$, sodium hydroxide $(\mathrm{NaOH})$, hydrogen peroxide $\left(\mathrm{H}_{2} \mathrm{O}_{2}\right)$, sulfuric acid $\left(\mathrm{H}_{2} \mathrm{SO}_{4}\right)$, sodium nitrite $\left(\mathrm{NaNO}_{3}\right)$, potassium permanganate $\left(\mathrm{KMnO}_{4}\right)$, polyvinylidene fluoride (PVDF) and hydrochloric acid $(\mathrm{HCl})$ were purchased from Sigma Aldrich and used as received. 


\subsection{Preparation of Hafnium Oxide/Graphene Oxide (HO)/(GO)} Nanocomposites

Hafnium oxide (HO) Nanoparticles were synthesized using precursor of hafnium tetrachloride $\left(\mathrm{HfCl}_{4}\right)$ and sodium hydroxide $(\mathrm{NaOH})$ by simple precipitation method. Initially, $0.4 \mathrm{M}(100 \mathrm{~mL})$ aqueous solution of $\mathrm{NaOH}$ was slowly added drop wise into $0.1 \mathrm{M}(100 \mathrm{~mL})$ aqueous solution of $\mathrm{HfCl}_{4}$ and subjected to continuous stirring using teflon coated magnetic bar for $24 \mathrm{~h}$, which gives a white coloured precipitate containing hafnium hydroxide. The $\mathrm{Hf}(\mathrm{OH})_{4}$ precipitate was washed thoroughly with millipore water and centrifuged at $2000 \mathrm{rpm}$ for 10 minutes to remove the residuals. This process was repeated many times until the precipitate was free from any trace impurities. Finally, the obtained product was dried in a hot air oven at $100^{\circ} \mathrm{C}$ for $24 \mathrm{~h}$ and further calcined at $800^{\circ} \mathrm{C}$ for $4 \mathrm{~h}$ which results in the formation of $\mathrm{HfO}_{2} \mathrm{NPs}$ [22].

Graphene oxide was synthesized from graphite powder by a Hummers method [23] and the detailed experimental conditions are reported earlier [24]. The HO/GO nanocomposites were prepared by mixing aqueous solution of GO into aqueous solution of HO at different ratios of GO $(20,30$ and $40 \mathrm{wt} \%$ ) to form the $\mathrm{HO} / \mathrm{GO}$ nanocomposites, then the mixture was heated at $100{ }^{\circ} \mathrm{C}$ for $12 \mathrm{~h}$. The resulting black precipitate was separated from the aqueous supernatant by centrifugation $2000 \mathrm{rpm}$ for $15 \mathrm{~min}$. Then the precipitate was dried at $100^{\circ} \mathrm{C}$.

\subsection{Electrode Preparation}

Prepared electrode material (HO, GO, HO/GO (30 wt\%) and HO/GO (40 wt\%), activated carbon and PVDF binder were mixed together in the ratio of 85:5:10 (wt\%) were deposited onto a platinum foil $\left(\mathrm{A}=0.5 \mathrm{~cm}^{2}\right)$. The active mass is around $400 \mu \mathrm{g}$.

\subsection{Instrumentation}

\subsubsection{X-Ray Diffraction}

The crystal structure, orientation and crystalline size of the HO/GO nanocomposites were examined by Rigaku X-ray diffractometer using $\mathrm{Cu}$ $\mathrm{k}_{\alpha}(\lambda=1.5406 \AA)$ radiation

\subsubsection{UV/Vis Spectroscopy}

The absorption spectra were recorded using Perkin-Elmer Lambda $\mathrm{UV} / \mathrm{vis} 35$ spectrophotometer in plastic cuvettes with an optical path of 10 $\mathrm{mm}$. The wavelength range was $200-800 \mathrm{~nm}$.

\subsubsection{Scanning Electron Microscopy (SEM)}

SEM images were taken using a scanning electron microscope VEGA3 TESCAN (Germany) equipped with a thermal field emission emitter and two different detector.

\subsubsection{Transmission Electron Microscopy (TEM)}

TEM images were obtained using Hitachi H-7650 (Singapore) microscope at maximum acceleration voltage of $120 \mathrm{keV}$.

\subsubsection{Electrochemical Measurements}

Cyclic Voltammetry (CV) experiments were performed using a CHI 661 C. The electrochemical cell consisted of $\mathrm{HO} / \mathrm{GO}$ as the working electrode, Standard calomel electrode (SCE) as the reference electrode, and platinum (Pt) wire as the counter electrode. Cyclic voltammetric measurements were performed using aqueous $1 \mathrm{M} \mathrm{H}_{2} \mathrm{SO}_{4}$ solution at ambient conditions. All electrochemical experiments were performed at room temperature.

The supercapacitor behavior of $\mathrm{HO} / \mathrm{GO}$ was investigated using a threeelectrode system comprised of HO/GO nanocomposites as the working electrode, Pt foil as the counter electrode and SCE as the reference electrode. The $\mathrm{CV}$ curves were recorded from 0 to $0.8 \mathrm{~V}$ with a scan rate of $10 \mathrm{mVs}^{-1}$.

The specific capacitance (C) was calculated by integrating the area under the $\mathrm{CV}$ curve according to Eq.(1):

$$
C s=\frac{1}{m v(V U-V L)} \int_{V L}^{V U} I(V) d V
$$

where $\mathrm{m}$ is the mass of active material $(\mathrm{g}), \mathrm{v}$ is the scan rate $\left(\mathrm{mVs}^{-1}\right), \mathrm{V}_{\mathrm{U}}$ and $V_{L}$ are the upper and lower voltage limits $(\mathrm{V})$ and $I$ is the current $(\mathrm{A})$.

The specific capacitance was in addition calculated from the slope of the galvanostatic charge-discharge curve at the different densities using the following Eq.(2) [25].

$$
C s=\frac{I \Delta t}{m \Delta V}
$$

where $\mathrm{I}$ is the discharge current $(\mathrm{mA}), \mathrm{m}$ is the mass of the electro-active material $(\mathrm{mg}): \Delta \mathrm{t}$ is the discharge time $(\mathrm{t})$.

https://doi.org/10.30799/jnst.118.18040304

\section{Results and Discussion}

3.1 Preparation and Characterization of Hafnium Oxide/Graphene Oxide (HO/GO) Nanocomposites

The preparation method of HO/GO nanocomposites is given in Fig. 1. First, hafnium oxide was synthesized which was reported by Ramadoss et al. [26]. Second, graphene oxide was prepared using a Hummers method. Third, the aqueous solution of $\mathrm{GO}$ is mixed with aqueous solution $\mathrm{HO}$ at three different ratios of GO content $(20,30$ and $40 \mathrm{wt} \%)$. The resulting three different ratios of $\mathrm{HO} / \mathrm{GO}$ nanocomposites were tested.

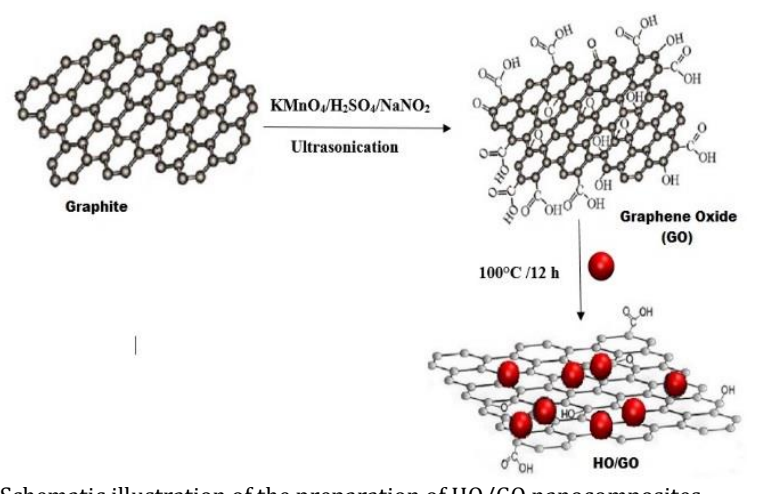

Fig. 1 Schematic illustration of the preparation of HO/GO nanocomposites

X-Ray Diffraction (XRD) analysis were performed on GO and HO before and after its reaction with each other to gain further knowledge on its crystal structure, purity and particle size. The XRD patterns of pure HO, GO and HO/GO nanocomposites at three different ratios were shown in Fig. 2. The crystalline structures of $\mathrm{HO}, \mathrm{GO}, \mathrm{HO} / \mathrm{GO}(20 \%), \mathrm{HO} / \mathrm{GO}(30 \%)$ and HO/GO (40\%) samples were studied by XRD. The XRD spectrum of HO (Fig. 2b) exhibited a sharp diffraction peak at $\left(2 \theta=24.55^{\circ}\right)$ with preferential orientation of the (-111) plane. The mean particle size of HO can be found using the Debye Scherer's formula, with the help of full width half-maximum of the peaks belongs to $\mathrm{HO}$ and is found to be nearly $\approx 20$ $\mathrm{nm}$. All the peaks of $\mathrm{HO}$ obtained in the XRD pattern were exactly matched with the monoclinic HO phase (JCPDS card No: 06-0318). The XRD pattern of GO (Fig. 2a) exhibited a strong and broad peak at $\left(2 \theta=23.39^{\circ}\right)$ which is compatible with the literature data (JCPDS, 75-2078) degree corresponding to d spacing of $3.842 \AA$ which might be attributed to very thin GO layer due to high degree of exfoliation. However, the apparent diffraction peak of GO sheets was discerned in the XRD pattern of HO/GO (20\%, 30\% and $40 \%)$ composites, this suggests that the layer-stacking regularity of GO sheets would be disrupted and low fraction of GO sheets did not influence the lattice structure of $\mathrm{HO}$ crystal, which is similar to that of $\mathrm{HO} / \mathrm{GO}$ composites.
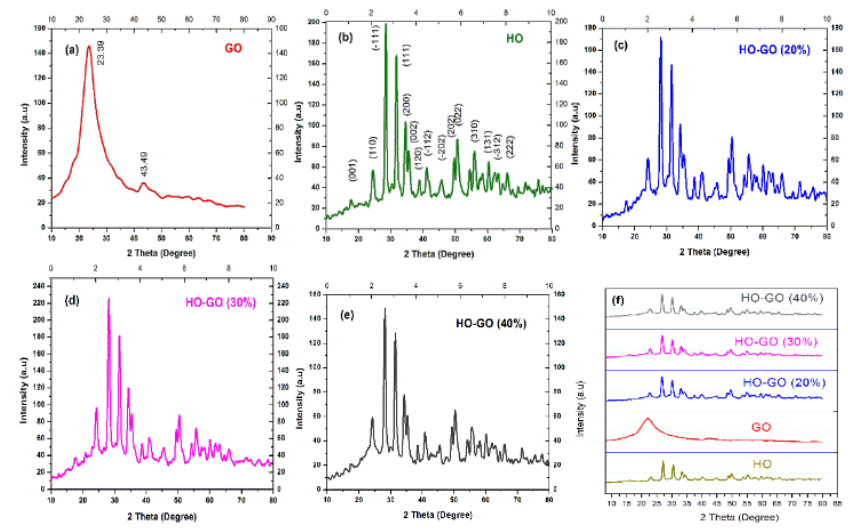

Fig. 2 XRD pattern of (a) GO, (b) HO, (c) HO/GO(20 wt\%), (d) HO/GO (30 wt \%), (e) HO/GO (40 wt\%) and f) all the samples (Comparison)

The UV-vis absorption spectra of HO, GO and HO/GO nanocomposites are depicted in Fig. 3. The absorption peak at $205 \mathrm{~nm}$ can be assigned to the absorption of HO [27]. GO dispersed in water exhibits a maximum absorption at $226 \mathrm{~nm}$, attributed to the $\pi-\pi *$ transition resulting from $\mathrm{C}=\mathrm{C}$ bonds from carboxylic acid functions. The absorption peak at $270 \mathrm{~nm}$ [28] is attributed to $\mathrm{n}-\pi *$ transition of aromatic C-O bonds i.e. graphene oxide (GO). The spectrum of $\mathrm{HO} / \mathrm{GO}$ nanocomposite varies significantly with different wt $\%$ of HO/GO employed. The absorption band at $196 \mathrm{~nm}$ is relatively small for $\mathrm{HO} / \mathrm{GO}(20 \%)$, but is significantly increased for higher ratios $206 \mathrm{~nm}$ for $\mathrm{HO} / \mathrm{GO}(30 \%)$ and $214 \mathrm{~nm}$ for $\mathrm{HO} / \mathrm{GO}$ (40\%). 

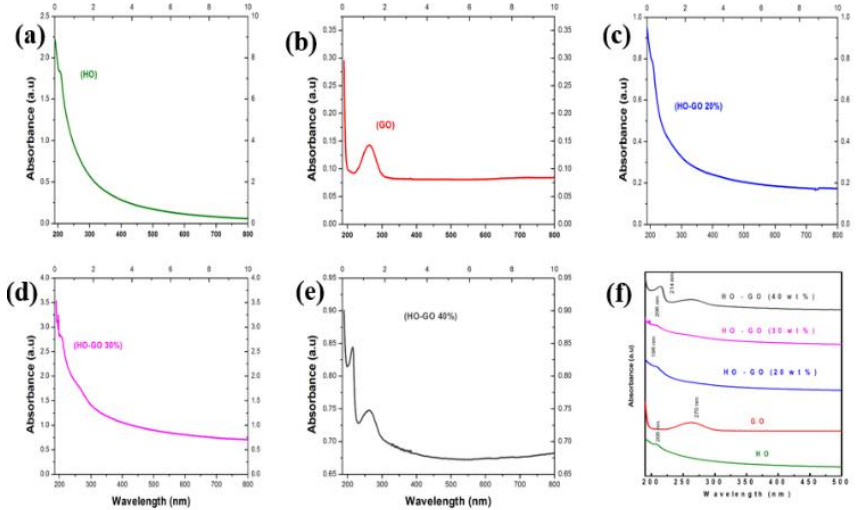

Fig. 3 (a-e) UV absorption spectrum of GO, HO, HO/GO(20 wt\%), HO/GO (30 wt\%) and $\mathrm{HO} / \mathrm{GO}(40 \mathrm{wt} \%)$ respectively and (f) UV absorption spectrum of all the samples (Comparison)
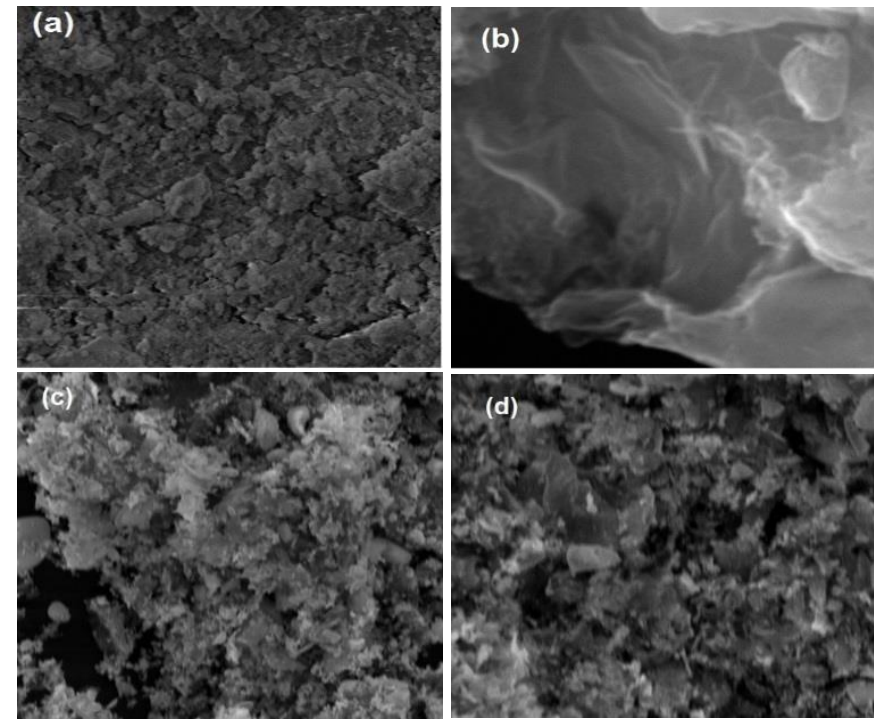

Fig. 4 (a-d) SEM images of HO, GO, HO/GO (30 wt\%) and HO/GO (40 wt\%) respectively
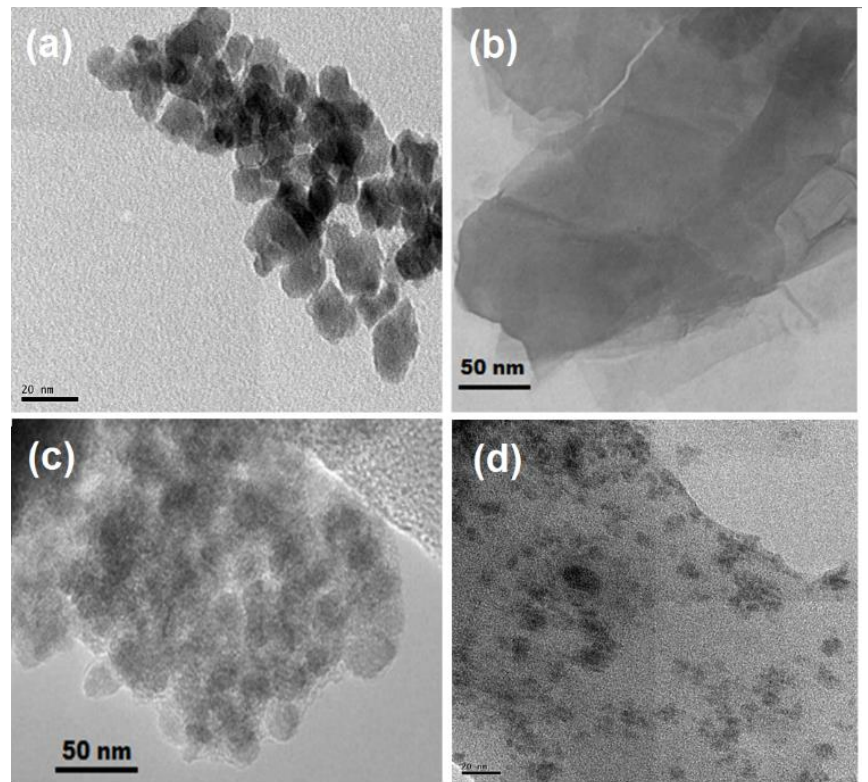

Fig. 5 (a-d) TEM images of HO, GO, HO/GO (30 wt\%) and HO/GO (40 wt\%) respectively

Fig. 4 shows the SEM images of pure HO, GO and HO/GO composites. Pure HO is possess a uniform distribution of dense particles spherical in shape (Fig. 4a). From the Fig. $4 \mathrm{~b}$ it is clearly revealed that the GO has a two dimensional sheet-like structure, also it is evident that GO has a multiple lamellar layer structure and it is possible to distinguish the edges of individual sheets from the SEM images. The films are stacked one above the other and also show wrinkled areas. It can also be noted that the GO sheets were thicker at the edges, why because the oxygen-containing functional groups were mainly combined at the edges of GO [29]. It can be observed from the SEM image (Figs. 4c and d) that the GO sheets are covered the surface of $\mathrm{HO}$ particles.

For further characterize, the morphology of the HO, GO and HO/GO composites, TEM were investigated. Samples were prepared by dipping carbon mesh grids into the ultrasonicated suspension and drying them in air. The TEM investigations shown in Fig. 5 revealed that the HO densely bound on the surface of the GO sheets, as it can be seen from TEM images, the average size of the $\mathrm{HO}$ particles were in the range of $20 \mathrm{~nm}$, which was in good agreement with XRD measurements. The EDS analysis suggests that the presence of hafnium (Hf), carbon (C) and oxygen (O) elements in the HO/GO nanocomposites (Fig. 6).

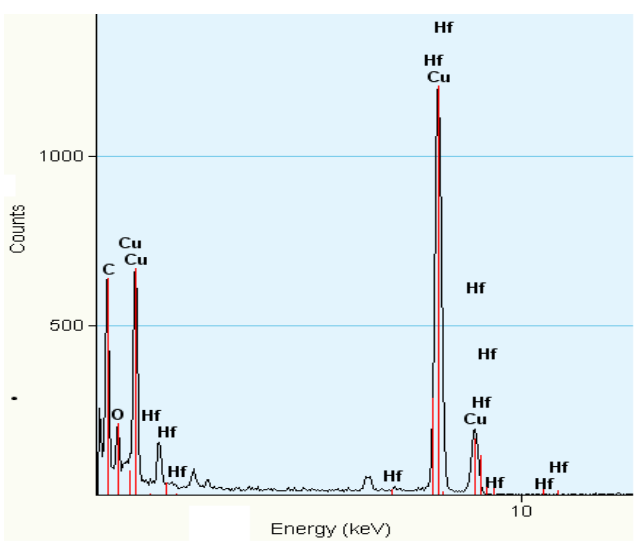

Fig. 6 EDS Spectrum of HO/GO nanocomposites

\subsection{Electrochemistry of Hafnium Oxide/Graphene Oxide Nanocomposites}

The electrochemical activity of the HO/GO nanocomposites was studied by measuring cyclic voltammetry and galvanostatic charge-discharge cycles of these nanomaterials. Full range $\mathrm{CV}$ of all the samples were scanned on CHI $661 \mathrm{C}$ electrochemical workstation. All the electroactive material show I/V compliance in one anodic and one cathodic step; redox transition between a semiconducting state and a conducting state are responsible for cathodic and anodic peaks. With scan rate, all such I/V compliances were found to consist of increasing peak current and a shift in the voltage to higher values. In a $\mathrm{CV}$, the higher the redox peak, the greater is the electrochemical reaction activity [30].

CV curves of all the electrodes clearly shows redox reactions, which exhibits anodic peaks associated with oxidation of material and a cathodic peak corresponding to reduction (Fig. 7). All of the among nanocomposites $\mathrm{HO} / \mathrm{GO}$ (40 wt\%) had the greatest redox activity while the redox activity is the least for $\mathrm{HO}$.
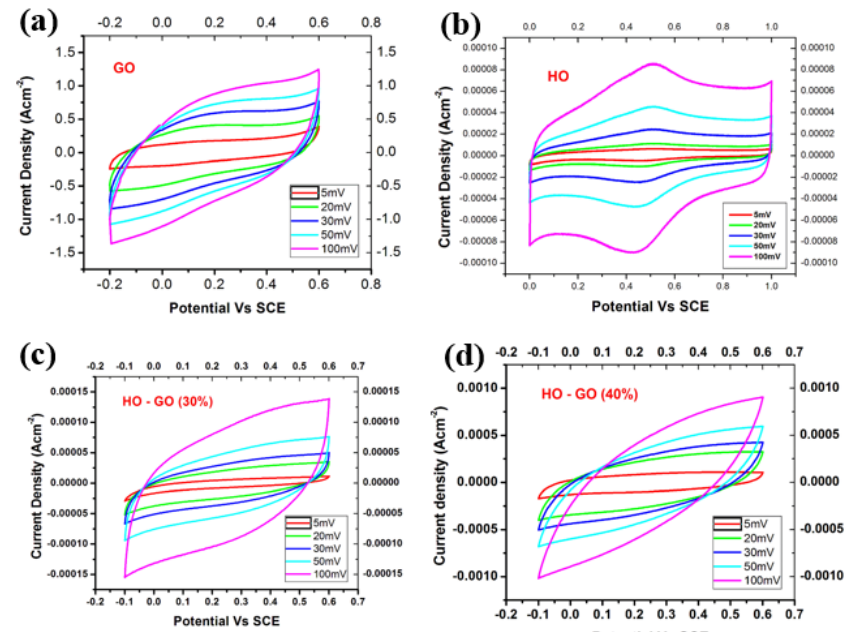

Fig. 7 Cyclic voltametric curves of a) HO, b) GO, c) HO/GO nanocomposite (30\%) and d) HO/GO nanocomposite $(40 \%)$

\subsection{Super Capacitive Characterization}

To check if the observed electrochemical conductivity is elevated enough to enable current collection, we investigated the supercapacitor behavior of the $\mathrm{HO} / \mathrm{GO}$ (30 and $40 \mathrm{wt} \%$ ) composite electrodes. In contrast to conventional high surface area materials such as conducting polymers and nonporous electrode materials like carbon nanotubes, the effective 
surface area of graphene and its derivatives is not solely dependent on the distribution of pores in the solid state [31]. The presence of oxygen functional groups enhances generally the wettability and the capacitance values of graphene-based supercapacitor [32]. Graphene oxide is known to comprise of well capacity and a potential material to store energy [33]. Two mechanisms are responsible for the specific capacitance of metal oxide electrode: the surface adsorption/desorption and the intercalation/deintercalation of alkali metal ions [34]. Fig. 7 shows the cyclic voltammograms of the different interfaces in $1 \mathrm{M} \mathrm{H}_{2} \mathrm{SO}_{4}$. Beside the HO/GO (40 wt\%) composite (Fig. 7d), rectangle-like shaped curves characteristic of capacitive behavior are observed with specific capacitance varying between $66 \mathrm{Fg}^{-1}$ and $268 \mathrm{Fg}^{-1}$, depending on the HO/GO nanocomposite (Table 1). The rectangular shape indicates that the main contribution to the capacitance is the charge and discharge of the double layer, which is enhanced by the presence of $\mathrm{HO}$ working probably as spacers. We observed that the specific capacitance for both electrodes decreased with an increase in the scan rate from $5 \mathrm{mVs}^{-1}$ to $100 \mathrm{mVs}^{-1}$. This is a common phenomenon and is caused by the insufficient time available for ion diffusion and adsorption inside the smallest pores within a large particle at high scan rate [35]. Similar results have been reported for GO/MO (Manganese oxide) nanocomposite with a specific capacitance of $211.2 \mathrm{Fg}^{-1}$ at a scan rate of $5 \mathrm{mVs}^{-1}$ [36]. Qi wang et al. [37] reported reduced graphene oxide (rGO)/ (ND) nanodiamond with 10/1 ratio showed the best performance with a specific capacitance of $186 \pm 10 \mathrm{Fg}^{-1}$ at scan rate $10 \mathrm{mVs}^{-1}$.

S. Xiang et al. [38] reported $\mathrm{TiO}_{2}$ and graphene nanocomposites revealed a maximum specific capacitance $84 \mathrm{Fg}^{-1}$ at a scan rate of $10 \mathrm{mVs}-$ 1. Graphene $/ \mathrm{TiO}_{2}$ nanocomposite exhibited a high specific capacitance of $165 \mathrm{Fg}^{-1}$ at a scan rate of $5 \mathrm{mVs}^{-1}$ in $1 \mathrm{M} \mathrm{Na}_{2} \mathrm{SO}_{4}$ electrolyte solution reported by Ramadoss et al. [39]. $\mathrm{RuO}_{2}$ /graphene, $\mathrm{TiO}_{2}$ /graphene and $\mathrm{Fe}_{3} \mathrm{O}_{4}$ /graphene nanocomposites observed a maximum specific capacitance of $265 \mathrm{Fg}^{-1}, 60 \mathrm{Fg}^{-1}$ and $180 \mathrm{Fg}^{-1}$ respectively with $1 \mathrm{M} \mathrm{H}_{2} \mathrm{SO}_{4}$ as the electrolyte at scan rate of $10 \mathrm{mVs}^{-1}$ are reported by Mishra et al. [40]. The prepared HO/GO nanocomposite electrode, which exhibited a maximum specific capacitance value is $268 \mathrm{Fg}^{-1}$ at a scan rate $5 \mathrm{mVs}^{-1}$. The specific capacitance of $\mathrm{HO} / \mathrm{GO}$ nanocomposite is higher than that of above mentioned reported values.
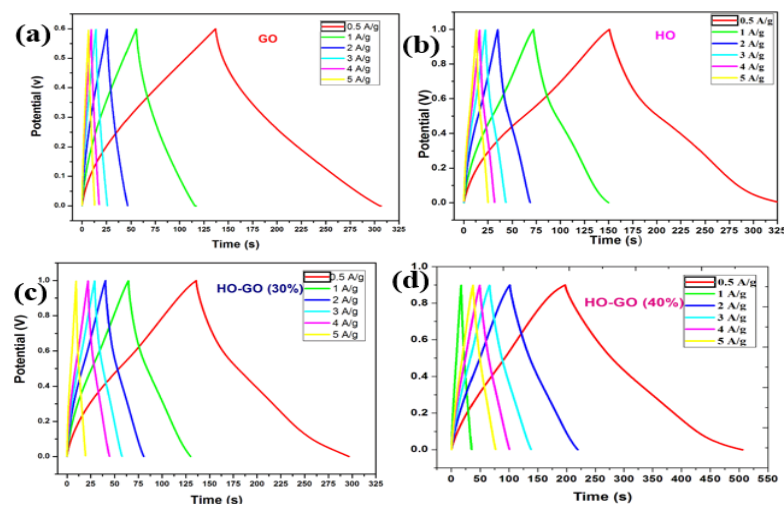

Fig. 8 Galvanostatic charge-discharge curve of the a) GO, b) HO, c), HO/GO nanocomposite (30 wt $\%$ ) and d) HO/GO (40 wt $\%$ )

To further evaluate the specific capacitance of our HO, GO and HO/GO nanocomposite electrode, galvanostatic charge-discharge measurements (Fig. 8) were performed in the current densities of $\left(0.5 \mathrm{Ag}^{-1}\right.$ to $\left.5 \mathrm{Ag}^{-1}\right)$. It can be seen that all of the curves were linear and exhibited a typical triangular shape, which revealed good electrochemical capacitive characteristics. The specific capacitance of the electrode can also be calculated from the galvanostatic charge-discharge curve at different densities using the Eq.(2).

Table 1 Specific capacitance of HO, GO, HO/GO (30 wt\%) and HO/GO (40 wt\%) nanocomposites using cyclic voltammetry with different voltage sweep rates and using the galvanostatic charge-discharge technique

\begin{tabular}{|c|c|c|c|c|c|c|c|c|c|c|c|}
\hline \multirow{3}{*}{ Product } & \multirow{2}{*}{\multicolumn{5}{|c|}{$\begin{array}{l}\text { Specific Capacitance }\left(\mathrm{Fg}^{-1}\right) \\
\text { (Cyclic Voltammetry) } \\
\text { Voltage Scan Rate }\left(\mathrm{mVs}^{-1}\right)\end{array}$}} & \multicolumn{6}{|c|}{$\begin{array}{l}\text { Specific Capacitance }\left(\mathrm{Fg}^{-1}\right) \\
\text { (Galvanostatic) }\end{array}$} \\
\hline & & & & & & Curr & nt De & sity ( & $\left.\mathrm{g}^{-1}\right)$ & & \\
\hline & $\overline{5}$ & 20 & 30 & 50 & 100 & 0.5 & 1 & 2 & 3 & 4 & 5 \\
\hline$\overline{\mathrm{HO}}$ & 147 & 134 & 128 & 123 & 118 & 91 & 78 & 69 & 62 & 56 & 53 \\
\hline GO & 200 & 131 & 118 & 107 & 94 & 145 & 102 & 73 & 42 & 40 & 37 \\
\hline $\begin{array}{l}\mathrm{HO} / \mathrm{GO} \\
(30 \mathrm{wt} \%)\end{array}$ & 231 & 128 & 95 & 62 & 34 & 156 & 121 & 117 & 109 & 98 & 83 \\
\hline $\begin{array}{l}\mathrm{HO} / \mathrm{GO} \\
\text { ( } 40 \mathrm{wt} \%)\end{array}$ & 268 & 171 & 137 & 103 & 66 & 165 & 133 & 127 & 117 & 115 & 93 \\
\hline
\end{tabular}

\section{Conclusion}

The present study shows that the $\mathrm{HO} / \mathrm{GO}$ hybrid nanostructure as an electrode material for supercapacitors was successfully synthesized using a simple mixing method. The surface morphology, microstructure, composition and capacitive behaviors of the prepared nanocomposite were well investigated. SEM and TEM images confirmed the uniform distribution of HO nanoparticles on the surface of GO sheets. While XRD confirm the nanosized materials with layered matrix. Our results showed that the HO/GO hybrid electrode exhibited a maximum specific capacitance of $268 \mathrm{Fg}^{-1}$ at a scan rate of $5 \mathrm{mVs}^{-1}$ in $1 \mathrm{M} \mathrm{H}_{2} \mathrm{SO}_{4}$ electrolyte solution. The remarkable performance of $\mathrm{HO} / \mathrm{GO}$ nanocomposite could be credited to the nanosize and surface area of $\mathrm{HO}$ particles along with good electric conductivity of GO. Moreover, the material exhibited excellent electrochemical stability (long cycle stability) for specific capacitance. These results suggest that $\mathrm{HO} / \mathrm{GO}$ hybrid electrodes are highly suitable, promising electrode for next generation high performance supercapacitor.

\section{Acknowledgement}

One of the author (Jayavel) is thankful to the Management, Meenakshi College of Engineering, West K.K. Nagar, Chennai-78 for providing laboratory facilities.

\section{References}

[1] L.L. Zhang, X.S. Zhao, Carbon-based materials as supercapacitor electrodes, Chem. Soc. Rev. 38(9) (2009) 2520-2531.

[2] D.N. Futaba, K. Hata, T. Yamada, T. Hiraoka, Y. Hayamizu, Y. Kakudate, Shapeengineerable and highly densely packed single-walled carbon nanotubes and their application as super-capacitor electrodes, Nat. Mater. 5(12) (2006) $987-$ 994.

[3] Y. Huang, J. Liang, Y. Chen, An overview of the applications of graphene-based materials in supercapacitors, Small 8(12) (2012) 1805-1834.

[4] P. Simon, Y. Gogotsi, Materials for electrochemical capacitors, Nat. Mater. 7 (2008) 845-854.

[5] H. He, J. Klinowski, M. Forster, A. Lerf, A new structural model for graphite oxide, Chem. Phys. Lett. 287 (1998) 53-56.

[6] A. Lerf, H. He M. Forster, J. Klinowski, Structure of graphite oxide revisited, J. Phys. Chem. B 102 (1998) 4477-4482.

[7] S. Stankovich, R.D. Piner, S.T. Nguyen, R.S. Ruoff, Synthesis and exfoliation of isocyanate-treated graphene oxide nanoplatelets, Carbon 44 (2006) 3342 3347

[8] J.K. Jeong, Y.P. Lee, R.J.W.E. Lahaye, M.H. Park, K.H. An, I.J. Kim, et al., Evidence of graphitic AB stacking order of graphite oxides, J. Am. Chem. Soc. 130 (2008) 1362-1366.

[9] F.G. Marcos, J.A. Rodriguez, Metal oxide nanoparticles, Brookhaven National Laboratory, USA, 2007.

[10] H. Mudila, S. Rana, M.G.H. Zaidi, Electrochemical performance of zirconia/graphene oxide nanocomposites cathode designed for high power density supercapacitor, Jour. Anal. Sci. Tech. 7(3) (2016) 1-11.

[11] Z.S. Wu, G. Zhou, L.C. Yin, W. Ren, F. Li, H.M. Cheng, Graphene/metal oxide composite electrode materials for energy storage, Nano Energy 1 (2012) 107131.

[12] Y.R. Lee, M.S. Song, K.M. Lee, I.Y. Kim, S.J. Hwang, Synthesis and electrochemical characterization of reduced graphene oxide-manganese oxide nanocomposites. J. Electrochem. Sci. Technol. 2(1) (2011) 1-7.

[13] H. Zhao, L. Pan, S. Xing, J. Luo, J. Xu, Vanadium oxides reduced graphene oxide composite for lithium-ion batteries and supercapacitors with improved electrochemical performance, J. Power Sources 222 (2013) 21-31.

[14] Y. Mai, F. Zhang, X. Feng, Polymer-directed synthesis of metal oxide containing nanomaterials for electrochemical energy storage, Nanoscale 6 (2014) 106121.

[15] C.M. Chen, Q. Zhang, J.Q. Huang, W. Zhang, X.C. Zhao, C.H. Huang, Chemically derived graphene-metal oxide hybrids as electrodes for electrochemical energy storage: pre-graphenization or post-graphenization, J. Mater. Chem. 22 (2012) 13947-13955.

[16] H. Yang, J. Jiang, W. Zhou, L. Lai, L. Xi, Y.M. Lam, Influences of graphene oxide support on the electrochemical performances of graphene oxide- $\mathrm{MnO}_{2}$ nanocomposites, Nanoscale Res. Lett. 6 (2011) 531-538.

[17] L. Harry, Tuller, Ionic conduction in nanocrystalline materials, Solid State Ionic 131 (2000) 143-157.

[18] R. Kotz, M. Carlen, Principles and applications of electrochemical capacitors. Electrochim. Acta 45 (2000) 2483-2496.

[19] K.H. An, W.S. Kim, Y.S. Park, Y.C. Choi, S.M. Lee, D.C. Chung, et al., Supercapacitors using single-walled carbon nanotube electrodes, Adv. Mater. 13 (2003) 497-500.

[20] Q. Wang, Z. Wen, J. Li, A Hybrid Supercapacitor fabricated with a carbon nanotube cathode and $\mathrm{TiO}_{2}$-B nanowire anode, Adv. Funct. Mater. 16 (2006) 2141-2146.

[21] Q. Ke, J. Wang, Graphene-based materials for supercapacitor electrodes - A review, Jour. Mater. 2(1) (2016) 37-54.

[22] A. Ramadoss, K. Krishnamoorthy, S.J. Kim, Novel synthesis of hafnium oxide nanoparticles by precipitation method and its characterization, Mater. Res. Bull. 147(9) (2012) 2680-2684. 
[23] W.S. Hummers Jr, R.E. Offeman, Preparation of graphitic oxide, J. Am. Chem. Soc. 80(6) (1958) 1339-1339.

[24] M.R. Das, R.K. Sarma, R. Saikia, V.S. Kale M.V. Shelke, P. Sengupta, Synthesis of silver nanoparticles in an aqueous suspension of graphene oxide sheets and its antimicrobial activity, Colloids Surf. B 83(1) (2011) 16-22.

[25] J. Yan, J. Liu Z. Fan, T. Wei, L. Zhang, High-performance supercapacitor electrodes based on highly corrugated graphene sheets, Carbon 50 (2012) 2179-2188.

[26] A. Ramadoss, K. Krishnamoorthy, S.J. Kim, Novel synthesis of hafnium oxide nanoparticles by precipitation method and its characterization, Mater. Res. Bull. 47(9) (2012) 2680-2684

[27] H. Takeuchi, D. Ha, T.J. King, Observation of bulk $\mathrm{HfO}_{2}$ defects by spectroscopic ellipsometry, Jour. Vac. Sci. Technol. A 22(4) (2004) 1337-1341.

[28] F.T. Thema, M.J. Moloto, E.D. Dikio, N.N. Nyangiwe, I. Kotsedi, M. Maaza, M. Khenfouch, Synthesis and characterization of graphene thin films by chemical reduction of exfoliated and intercalated graphite oxide, Jour. Chem. 2013 (2012) 1-6.

[29] A.N. Syed, S. Nidhi, K. Lailesh, Synthesis of graphene oxide by modified Hummers method and its thermal reduction to obtain reduced graphene oxide, Graphene 6 (2017) 01-18.

[30] H. Mudila, S. Rana, M.G.H. Zaidi, S. Alam, Polyindole/graphene oxide nanocomposites the novel material for electrochemical energy storage, Fullerenes Nanotubes Carbon Nanostruct. 23(1) (2015) 20-26.

[31] M.D. Stoller, S. Park, Y. Zhu, J. An, R.S. Ruoff, Graphene-based ultracapacitors, Nano Lett. 8(10) (2008) 3489-3502.
[32] J. Yang, S. Gunasekaran, Electrochemically reduced graphene oxide sheets for use in high performance supercapacitors, Carbon 51 (2013) 36-44.

[33] J.L. Reta, R. Garcia, G. Sandoval, J.L. Rivera, Preparation of zirconium oxides modified with borate ions tested in catalytic dehydration of ethanol, Rev. Mex. Defisicas. 55(1) (2009) 98-101.

[34] S. Lee, B. Kang, S. Lee, H. Jeong, I.A.C. Song, Optical, electrical, and structural properties of ultrathin zirconium oxide films, J. Korean Phys. Soc. 57(6) (2010) 1811-1815.

[35] W.W. Li, X.B. Yan, Q.J. Xue, Multilayer hybrid films consisting of alternating graphene and titanium dioxide for high performance specific capacitance, J. Matter. Chem. C 47 (2013) 1413-1422.

[36] C. Sheng, Z. Junwu, W. Xiaodong, H. Qiaofeng, W. Xin, Graphene oxide- $\mathrm{MnO}_{2}$ nanocomposites for supercapacitors, Am. Chem. Soc. 4(5) (2010) 2822-2830.

[37] Qi Wang, N. Plylahan, M.V. Shelke, R.R. Devarapalli, M. Li, P. Subramanian, et al., Nanodiamond particles/reduced graphene oxide composites as efficient supercapacitor electrodes, Carbon 68 (2013) 175-184.

[38] S. Xiang, X. Ming, W. Gongkai, S. Hongtao, S. Andrew, Cavanagh, et al., Atomic layer deposition of $\mathrm{TiO}_{2}$ on graphene for supercapacitors, Jour. Electrochem. Soc. 159(4) (2012) A364-A369.

[39] A. Ramadoss, S.J. Kim, Improved activity of graphene- $\mathrm{TiO}_{2}$ hybrid electrode in an electrochemical supercapacitor, Carbon 63 (2013) 434-445.

[40] A.K. Mishra, S. Ramaprabhu, Functionalized graphene-based nanocomposites for supercapacitor application, J. Phys. Chem. C 115 (2011) 14006-14013. 